\title{
Uma revisão crítica de três perspectivas sobre o desenvolvimento da escrita
}

\author{
A critical review of three perspectives \\ in spelling development
}

\author{
Tatiana Cury POLLO' \\ Rebecca TREIMAN ${ }^{2}$ \\ Brett KESSLER ${ }^{2}$
}

\section{Resumo}

Aprender a ler e a escrever está entre os maiores desafios de uma criança. Um componente importante da alfabetização é a escrita. Apesar do aumento de interesse pela escrita nos últimos anos, seu estudo ainda não atrai tanta atenção quanto o da leitura. Este artigo revisa três perspectivas no estudo do desenvolvimento da escrita: fonológica, construtivista e da aprendizagem estatística. Atenção especial é dada aos estudos que examinam o desenvolvimento da escrita de forma translinguística, já que são cruciais para diferenciar as propriedades do desenvolvimento da escrita que são universais, daquelas que são específicas ao sistema de escrita da criança. As tradicionais perspectivas fonológicas e construtivistas são revisadas e criticamente avaliadas sob a perspectiva da aprendizagem estatística, mediante a revisão de seus conceitos fundamentais, com base estudos recentes que corroboram algumas de suas premissas.

Palavras-chave: Construtivismo; Escrita; Estatística.

\begin{abstract}
Learning how to read and spell can be one of the biggest challenges in children's lives. Although interest in spelling development has increased in recent years, the study of spelling has still not attracted as much attention as the study of reading. This article reviews three perspectives in the study of spelling development: phonological, constructivist and the statistical learning perspectives. We devote special attention to studies that have examined spelling development cross-linguistically, as these studies are crucial for differentiating universal properties of spelling development from those that are adaptations to specific features of the child's language or target writing system. The traditional phonological and constructivist perspectives will be reviewed and evaluated and a new perspective, statistical learning, will be introduced, along with a revision of its fundamental concepts and recent studies that corroborate its claims.
\end{abstract}

Keywords: Constructivism; Spelling; Statistics.

$\nabla \nabla \nabla v$

1 Universidade Federal de São João Del Rei, Departamento de Psicologia, Programa de Pós-Graduação em Psicologia. Praça Dom Helvécio, 74, Bairro Fábricas, 36301-170, São João Del Rei, MG, Brasil. Correspondência para/Correspondence to: T.C. POLLO. E-mail: $<$ tpollo@gmail.com>.

2 Washington University, Psychology Department, Graduate School of Arts and Sciences. St. Louis, MO, United States of America. 
Aprender a ler e a escrever está entre os maiores desafios de uma criança. Um componente importante da alfabetização é a escrita. Apesar do aumento de interesse pela escrita nos últimos anos, seu estudo ainda não atrai tanta atenção quanto o da leitura. Os estudos sobre a escrita merecem atenção especial por causa do interesse pedagógico em entender como as crianças assimilam essa faceta da alfabetização, além de conhecer a melhor maneira de guiá-los nesse caminho. Além disso, a escrita infantil fornece informações sobre o conhecimento das características gráficas e fonológicas da escrita, o que dificilmente seria obtido de outra forma.

Este artigo revisa três perspectivas no estudo do desenvolvimento da escrita: fonológica, construtivista e da aprendizagem estatística. Atenção especial será dada aos estudos que examinam o desenvolvimento da escrita de forma translinguística, já que são cruciais para diferenciar as propriedades do desenvolvimento da escrita que são universais, daquelas que são específicas ao sistema de escrita da criança.

\section{A perspectiva fonológica}

A perspectiva fonológica acredita que o maior desafio das crianças, quando estão aprendendo a escrever num sistema de escrita alfabético, é o entendimento de que as letras representam fonemas (Liberman, Shankweiler, Fischer, \& Carter, 1974). As crianças também precisam possuir conhecimento alfabético ou conhecimento de correspondências letra-som específicas. Porém, analisar a fala em fonemas parece ser mais difícil, nesse paradigma, do que aprender a ligação entre fonemas e letras.

A teoria fonológica descreve o desenvolvimento da escrita como uma habilidade, cada vez maior, de mapear os sons das palavras relacionando-os às letras fonologicamente apropriadas (Gough \& Hillinger, 1980). Teóricos como Ehri (1998), Frith (1985), Henderson (1985) e Gentry (1982) representam essa perspectiva. Eles propõem modelos de fases ou estágios que diferem em aspectos importantes, mas que aqui serão abordados conjun- tamente por enfatizarem a aquisição do conhecimento fonológico durante a alfabetização. O termo "etapa" será utilizado de modo genérico, distinguindo-se os termos "fase" e "estágio". Assim, as crianças seguem por uma etapa inicial, em que as escritas não são fonológicas, até a etapa final, quando elas são fonologicamente apropriadas. A seguir, apresenta-se uma breve revisão de duas teorias representativas: a teoria de estágios de Gentry (1982), que propõe um modelo baseado, em parte, num estudo de caso sobre uma criança que aprendeu a escrever espontaneamente (Bissex, 1980); e, após, a teoria de fases proposta por Ehri (1998).

Teóricos do paradigma fonológico argumentam que, em um primeiro momento, na fase pré-alfabética (Ehri, 1991) ou estágio pré-comunicativo (Gentry, 1982), as escritas das crianças não têm relação com os sons das palavras. Por exemplo, uma criança americana escreveu quick (rápido) com 〈HS〉 (Ehri, 1991), uma escrita pré-fonológica, já que as letras $\langle H\rangle$ e $\langle S\rangle$ não têm nenhuma relação com os sons da palavra.

Quando as crianças aprendem os nomes das letras, elas começam a entender que letras representam sons; então, representam alguns sons das palavras com as letras apropriadas. Bissex (1980) e Gentry (1982) citam o exemplo de uma criança de cinco anos de idade, que, tentando obter atenção da mãe, escreve 〈RUDF〉 para Are you deaf? (Você está surda?). Para esse tipo de escrita, dá-se o nome de escrita alfabética parcial ou semifonética. Como os exemplos de 〈R〉 para are e 〈U〉 para you demonstram, as representações de sons que as crianças utilizam podem refletir os nomes das letras do alfabeto. Estudos demonstram que crianças familiarizadas com os nomes das letras utilizam esse conhecimento para guiá-las em suas escritas iniciais. Nomes das letras são frequentes nas palavras de alguns idiomas (Pollo, Kessler, \& Treiman, 2005), e as crianças podem escrever apenas a letra, utilizando a estratégia de nomes das letras (Treiman, 1994). Por exemplo, crianças americanas podem escrever car (carro) como 〈CR〉, usando a consoante 〈R〉 para escrever todos os sons do nome dessa letra (International Phonetic Association, 1999). Como os nomes das vogais coincidem com os sons que 
elas representam, a evidência do uso de vogais como nome de letras é mais indireta. Treiman (1993) demonstrou a importância dos nomes das vogais ao observar que crianças americanas tendem a representar nomes de vogais quando escrevem um som de vogal idêntico ao nome da letra.

A estratégia de nome das letras tem sido documentada não só em crianças americanas que falam inglês, mas também em aprendizes de outras línguas, como o hebraico (Levin, Patel, Margalit, \& Barad, 2002) e o português (Cardoso-Martins, Resende, \& Rodrigues, 2002). Em culturas em que a aprendizagem dos nomes das letras não é enfatizada, como a Inglaterra, esses efeitos são fracos ou inexistentes (Ellefson, Treiman, \& Kessler, 2009).

A próxima etapa, chamada de fase alfabética total ou estágio fonético, surge quando as crianças produzem escritas que representam totalmente a fonologia das palavras. Crianças podem escrever corretamente várias palavras, como 〈CAR〉 para car 'carro' ou prover uma escrita fonologicamente plausível, como 〈KAR〉. Nesse estágio, a maioria dos fonemas é representada nas escritas das crianças.

De forma consistente com a perspectiva fonológica, muitos pesquisadores demonstram que as escritas iniciais das crianças são em grande parte uma tentativa de representar os sons nas palavras (Read, 1986; Treiman, 1993). Esses pesquisadores relatam que, quando não sabem escrever certos sons, as crianças inventam suas próprias escritas, a chamada escrita inventada.

O trabalho pioneiro sobre escritas inventadas foi elaborado por Read (1986). Suas observações sobre escritas precoces questionaram a visão tradicional de que crianças aprendem a escrever por meio de memorização, deslocando o foco para o aspecto criativo das escritas infantis. Os erros de escrita mais comuns entre as crianças refletem algumas diferenças fonéticas que passam despercebidas aos adultos, talvez por estarem eles mais expostos às escritas corretas.

Como ilustrado, no estágio fonético, as crianças escolhem as letras pelos sons, sem nenhuma preocupação ortográfica convencional. Isto é, as crianças menores ainda não conhecem os pa- drões grafotáticos de seu sistema de escrita, como as restrições na ordem ou organização das letras, a exemplo da norma que veda a presença de rr ou ss no início das palavras. Quando as crianças começam a aprender sobre escritas convencionais e padrões de escritas, elas entram no estágio de transição (Gentry, 1982). Finalmente, alcançam o estágio correto (Gentry, 1982) ou a fase alfabética consolidada (Ehri, 1998), quando se tornam leitores e escritores competentes.

É grande a importância da teoria fonológica no estudo do desenvolvimento da escrita. Um dos pontos positivos dessa perspectiva está no reconhecimento de que crianças utilizam conhecimento linguístico em suas escritas. Essa perspectiva se opõe duramente ao conceito de que aprender a escrever é puramente um processo de memorização. Os trabalhos que seguem o ponto de vista fonológico foram precursores do conceito contemporâneo de que erros infantis refletem conhecimento sobre as propriedades sonoras das palavras. Estudos sobre o desenvolvimento da escrita mostram que a teoria fonológica, por si só, é capaz de explicar uma ampla gama de fenômenos. Outro aspecto positivo dessa abordagem é a utilização tanto de dados naturalísticos quanto experimentais. Essa combinação reúne a validade ecológica de observações naturalísticas ao rigor quantitativo de experimentos. A teoria fonológica tem um papel fundamental na alfabetização, já que tem orientado educadores e incentivado professores a analisarem a fala em fonemas e a ensinarem as correspondências letra-som.

Como a maior parte do trabalho na perspectiva fonológica tem estudado o idioma inglês, surge a questão da generalização desse modelo em alfabetizandos de outras línguas. Wimmer e Hummer (1990) sugeriram que, quando as crianças aprendem a escrita em sistemas com correspondências letra-som mais regulares, como a língua alemã, elas pulam as fases iniciais e vão direto à fase alfabética completa. No entanto, existe evidência para as fases pré-fonética e fonética de desenvolvimento da escrita, mesmo no português, em que as correspondências letra-som são mais regulares do que em inglês (Cardoso-Martins, 2005; Cardoso-Martins, Corrêa, Lemos, \& Napoleão, 2006). 
Uma característica dos sistemas de escrita que recebe atenção especial na teoria fonológica é a regularidade das relações entre os sons e as letras. Alguns estudos têm mostrado que esse é um fator importante na alfabetização (Defior, Martos, \& Cary, 2002; Seymour, Aro, \& Erskine, 2003). Nos sistemas de escrita regulares, as crianças aprendem a ler e a escrever melhor, mais rápido e com menos erros do que as crianças alfabetizadas em sistemas de escrita mais irregulares. Várias descobertas sugerem que o desenvolvimento da escrita é mais lento em inglês do que em sistemas de escrita mais regulares, como o tcheco (Caravolas \& Bruck, 1993) e o alemão (Wimmer \& Landerl, 1997). As diferenças encontradas entre o desenvolvimento da escrita em inglês e em outras línguas são atribuídas às diferenças das regularidades letra-som nesses sistemas de escrita (Caravolas, 2004). Contudo, a maior parte das diferenças não tem sido sistematicamente quantificada. As relações letra-som de outras línguas, além do inglês, têm atraído pouca atenção dos pesquisadores. Há necessidade de informações confiáveis e abrangentes sobre outros sistemas ortográficos, além de estudos sobre as características de outros idiomas que podem ser relevantes para as crianças em fase de alfabetização.

O maior inconveniente da perspectiva fonológica é que ela tende a dar pouca atenção aos aspectos não fonológicos da escrita. Pesquisadores dessa tradição acreditam que crianças possuem certas habilidades relacionadas com a alfabetização, incluindo o conhecimento sobre as formas das letras e nomes. O fato de que as crianças na fase pré-fonética (ou estágio pré-comunicativo) do desenvolvimento da escrita muitas vezes utilizam letras, ao invés de outros símbolos quando escrevem, sugere que elas já possuem algum conhecimento sobre o sistema de escrita. Não obstante, os pesquisadores que seguem a perspectiva fonológica não têm estudado com profundidade a escrita de crianças na fase pré-fonológica.

As produções de crianças pré-fonológicas que parecem ser sequências de letras aleatórias, por exemplo, podem consistir em letras do seu nome (Treiman, Kessler, \& Bourassa, 2001) ou refletir elas foram expostas, como as frequências de diferentes letras e grupos de letras (Kessler, Pollo, Treiman, \& Cardoso-Martins, 2013; Pollo, Kessler, \& Treiman, 2009). Quando as crianças começam a escrever fonologicamente, como a pesquisa sugere, elas continuam a mostrar alguma sensibilidade a padrões gráficos e sequências de letras admissíveis na escrita. Por exemplo, crianças americanas de cinco ou seis anos de idade já entendem que certas sequências de letras, como ck ou rr, raramente ocorrem no início das palavras. Evidência dessa sensibilidade precoce vem de escritas produzidas naturalmente (Treiman, 1993), bem como de estudos experimentais em que as crianças julgam que pseudopalavras, como baff, parecem mais com uma palavra verdadeira do que bbaf (Cassar \& Treiman, 1997). Em inglês, as consoantes normalmente não se repetem no início das palavras. Da mesma forma, crianças francesas da primeira série, com seis anos de idade, são sensíveis às letras que podem ser duplicadas (Pacton, Perruchet, Fayol, \& Cleeremans, 2001). Tais resultados sugerem que escritas precoces utilizam mais do que apenas a fonologia. O conhecimento não fonológico é importante desde o início do desenvolvimento da escrita e não está restrito às fases posteriores.

\section{A perspectiva construtivista}

Uma segunda teoria no estudo do desenvolvimento da escrita é a chamada perspectiva construtivista, muito influente em países de línguas românicas: francês (Besse, 1996), italiano (Pontecorvo \& Zuchermaglio, 1988), português (Silva \& Alves-Martins, 2002) e espanhol (Ferreiro \& Teberosky, 1982).

Os pesquisadores da tradição construtivista foram influenciados pela obra de Piaget, que criou um método de observação clínica para estudar como as crianças entendem o mundo. O autor postulou uma teoria geral de estágios de desenvolvimento, que mais tarde foi aplicada a uma variedade de comportamentos específicos, incluindo a leitura e a escrita. Ferreiro (1990); Ferreiro e Teberosky (1982); Vernon e Ferreiro, (1999) foram particularmente influentes na ampliação do quadro 
de Piaget para o desenvolvimento da linguagem escrita. Eles estudaram as concepções infantis sobre a linguagem escrita, propondo que as crianças sabem muito sobre a escrita, antes mesmo de compreender o princípio alfabético.

Esses autores descreveram três estágios na evolução da escrita, em que as crianças adotam e abandonam diferentes hipóteses sobre a linguagem escrita até que entendam o princípio alfabético.

No primeiro estágio - pré-silábico -, as crianças ainda não entendem que a escrita representa os sons da linguagem oral, não obstante apresentem certas hipóteses sobre a linguagem escrita. Uma delas é o princípio da quantidade mínima, pelo qual as crianças acreditam que um texto precisa ter algumas letras. De acordo com Ferreiro e Teberosky (1982), esse mínimo é fixo para dada criança, tipicamente três ou quatro letras, e independente do número mínimo de letras das palavras. Nesse estágio, as crianças também acreditam que as letras de uma palavra devem ser diferentes umas das outras, o que se denomina hipótese da variação. Além disso, Ferreiro, Pontecorvo e Zucchermaglio (1996) sugeriram que a preferência das crianças pela variação é independente da frequência de letras repetidas nos sistemas de escrita a que estão expostas.

À medida que as crianças aprendem mais sobre a escrita, de acordo com Ferreiro e seus colegas, elas apresentam a hipótese de que as letras na escrita representam sílabas. Isso resulta em escritas silábicas, em que as crianças escrevem um símbolo por sílaba. Por exemplo, as crianças podem escrever o dissílabo sapo 'sapo' como 〈AO〉 (Ferreiro \& Teberosky, 1982). Relatos de escritas silábicas são frequentes não só em espanhol, mas também em outras línguas neolatinas, como o português (Nunes, Carraher, \& Rego, 1984) e o italiano (Pontecorvo, 1996).

O estágio silábico é crucial na teoria de desenvolvimento da escrita de Ferreiro e Teberosky (1982), já que é considerado a primeira tentativa da criança de representar na escrita os sons da fala. A partir do momento em que as crianças começam a ganhar mais experiência com a linguagem escrita, elas passam a observar que o número de letras ge- ralmente excede o número de sílabas. Isso faz que passem da escrita silábica para a escrita alfabética quando entendem que as letras representam sons menores que sílabas, ou seja, fonemas.

A teoria construtivista reconhece que crianças de sociedades letradas sabem muito sobre a escrita, antes mesmo de entenderem que ela representa a fala no nível dos fonemas. Nos países de línguas românicas, a teoria de Ferreiro é o paradigma predominante para explicar a aquisição da escrita por crianças. Porém, a despeito da sua popularidade e aceitação em países da América Latina, incluindo o Brasil, a teoria de Ferreiro apresenta algumas limitações.

Uma delas é a falta de evidência para o estágio silábico, o mais importante da teoria. Kamii, Long, M. Manning e Manning (1990) não encontraram evidência para um estágio silábico entre crianças que falam inglês. Em vez disso, relataram que as crianças representam palavras apenas com consoantes, o que os autores chamaram de estágio consonantal. Até mesmo nas línguas latinas, estudos recentes levantam dúvidas sobre a existência de um estágio silábico. Escritas como 〈AO〉 para sapo são muitas vezes apresentadas como escritas silábicas, já que a criança escreveu duas letras para uma palavra dissílaba. Contudo, nesse exemplo, é típico que as letras correspondam aos nomes das letras escutados nas palavras. Assim, as escritas podem, alternativamente, refletir o uso de nomes de letras pelas crianças (Cardoso-Martins et al. 2006; Pollo et al. 2005; Treiman \& Kessler, 2003).

A hipótese de quantidade mínima proposta pela teoria construtivista também foi questionada. Pollo et al. (2009) descobriram que as crianças não evitam escritas de apenas uma ou duas letras tão frequentemente quanto sugerido. Na verdade, a proporção de palavras de uma e duas letras nas escritas das crianças foi muito semelhante à proporção encontrada nos textos escritos dos respectivos idiomas. Crianças brasileiras usaram palavras com menos de três letras com mais frequência do que as americanas. Essa diferença reflete a desigualdade entre as línguas, já que o português contém maior número de palavras curtas do que o inglês. A ideia de que as crianças tendem a evitar a 
repetição de letras em uma palavra, a hipótese de variação, também parece ser mais covenientemente explicada pela exposição ao texto impresso. Como o inglês tem uma taxa de repetição maior do que o português, crianças americanas são mais expostas à repetição e tendem a usá-la mais frequentemente do que as brasileiras.

\section{Perspectiva da aprendizagem estatística}

A perspectiva da aprendizagem estatística concorda com a ideia de que crianças em sociedades letradas têm muitos conhecimentos sobre a escrita antes de entenderem que as letras representam fonemas. Os teóricos da aprendizagem estatística entendem que existem padrões significativos nas primeiras escritas das crianças. Não obstante, enquanto construtivistas tendem a enfatizar construções que emergem espontaneamente da mente da criança, a perspectiva da aprendizagem estatística discorda dessa visão e enfatiza que as escritas das crianças refletem características dos dados a que são expostas, filtradas pelos seus mecanismos de percepção e aprendizagem.

Nesse contexto, estatística refere-se a frequências. Um padrão ou uma regularidade estatística existe quando um grupo de eventos ocorre mais do que seria esperado pelo acaso. Existe evidência considerável de que crianças e adultos podem demonstrar aprendizagem implícita de regularidades estatísticas (Zacks \& Hasher, 2002). Saffran, Aslin e Newport (1996) têm demonstrado, por exemplo, que regularidades estatísticas auxiliam bebês, crianças e adultos a segmentarem palavras.

A aplicabilidade da perspectiva da aprendizagem estatística à escrita pode ser, em um primeiro momento, surpreendente. A ideia de que crianças prestam atenção ao texto impresso e aprendem implicitamente alguns de seus padrões parece contrastar com resultados de que crianças novinhas prestam atenção às figuras, e não ao texto, quando os adultos leem para elas (Evans \& Saint-Aubin, 2005). Entretanto, a perspectiva da aprendizagem estatística entende que crianças em sociedades letradas participam de muitas outras atividades que podem prover experiências com a linguagem escrita.
Ou seja, a maioria das crianças já tem contato, mesmo que implícito, com a linguagem escrita. Assim, as escritas iniciais das crianças podem refletir conhecimentos adquiridos pela exposição a esses materiais.

Uma faceta importante da perspectiva da aprendizagem estatística é que o mesmo mecanismo de aprendizagem é responsável pela aquisição da escrita. Espera-se, nessa perspectiva, que as crianças aprendam uma gama de informações simultaneamente, contrastando com a ideia de estágios diferentes. Para ilustrar esse padrão, pode-se dar o exemplo de como as crianças aprendem princípios grafotáticos, ou seja, princípios sobre restrições quanto à ordem e organização das letras. A criança pode aprender alguns desses princípios precocemente; por exemplo, saber quando utilizar ou não as letras maiúsculas. Mas outros padrões grafotáticos podem ser assimilados mais tardiamente. Isso contrasta com a perspectiva fonológica, segundo a qual o aprendizado grafotático ocorre ao final do desenvolvimento da escrita. Outra característica da perspectiva da aprendizagem estatística é que as estratégias iniciais das crianças são fortemente influenciadas pelas propriedades do sistema de escrita que estão aprendendo. Dessa forma, deve-se esperar produções bem distintas de crianças que falam idiomas diferentes, mesmo antes que comecem a produzir escritas fonologicamente apropriadas. Em outras palavras, as escritas iniciais das crianças não são universais.

Um alicerce importante da perspectiva da aprendizagem estatística é o modelo conexionista. Esse modelo apresenta um padrão simples, porém preciso, para explicar como as pessoas aprendem regularidades estatísticas, explicando a cognição em termos de redes de unidades simples. A aprendizagem de padrões envolve modificar as conexões entre as unidades, em resposta à exposição a um número considerável de exemplos (Seidenberg, 1997). Estudos recentes enfatizam que mecanismos de aprendizagem conexionistas captam regularidades sutis na entrada (dados sensoriais), fornecendo, então, melhor explicação para a linguagem escrita adulta do que modelos anteriores, que se focam em regras no estilo tudo ou nada. Nesse 
sentido, Hutzler, Ziegler, Perry, Wimmer e Zorzi (2004) argumentam que os modelos conexionistas são capazes de explicar a vantagem que existe no aprendizado de sistemas de escrita mais regulares. Modelos conexionistas têm sido desenvolvidos para explicar dados de escritas típicas e deficientes (Houghton \& Zorzi, 2003). Esses modelos carecem de mais desenvolvimento para simular como humanos escrevem, mas a ênfase na sensibilidade do aprendiz às propriedades de dados sensoriais fornece uma importante base para a perspectiva estatística.

Estudos têm demonstrado que padrões que adultos e crianças encontram em suas experiências cotidianas com o texto impresso influenciam sua escrita. Um padrão que exerce importante influência nas crianças desde cedo é o nome próprio. Desde muito novas, elas veem frequentemente a escrita de seus nomes, e tal exposição parece ter um papel central no progresso da escrita. Estudos demonstraram que crianças pequenas identificam as letras do próprio nome com mais precisão que outras letras (Levin \& Aram, 2004; Treiman \& Broderick, 1998; Treiman, Kessler, \& Pollo, 2006). Outros estudos têm demonstrado que as crianças mais novas tendem a usar excessivamente as letras do próprio nome na tentativa de escrever outras palavras (Pollo et al., 2009; Treiman et al., 2001).

Quando as crianças são expostas a um número maior de palavras impressas, o efeito da exposição aos próprios nomes parece ser proporcionalmente reduzido. As crianças começam a ser influenciadas por padrões mais gerais do sistema de escrita. Estudos que corroboram essa visão mostram que crianças pré-fonológicas são inclinadas a utilizar letras e grupos de letras mais comuns em sua língua escrita (Pollo et al., 2009). Mesmo crianças já fonológicas podem utilizar letras não fonologicamente plausíveis, tais como aquelas que são mais recorrentes nos materiais de leitura (Treiman et al., 2001).

Se propriedades das palavras impressas influenciam a escrita das crianças, certos fenômenos podem encontrar uma explicação mais parcimoniosa dentro da perspectiva da aprendizagem estatística. Essa perspectiva pode também explicar algumas similaridades e diferenças observadas entre crianças que foram expostas a dados linguísticos e ortográficos diferentes. Dessa forma, estudos quantitativos translinguísticos são cruciais para o melhor entendimento da alfabetização.

A seguir, discutem-se estudos que ilustram como o modelo da aprendizagem estatística pode explicar o desenvolvimento da escrita.

Pollo et al. (2005) investigaram a aquisição da escrita em português e inglês. Existem relatos sobre diferenças na forma como crianças que falam esses dois idiomas aprendem a escrever. Os autores investigaram se o modelo estatístico pode explicar tais diferenças. Uma diferença relatada entre os que escrevem em inglês e em português envolve a aquisição de consoantes e vogais. Enquanto crianças de línguas românicas são propensas a omitir as consoantes, produzindo escritas somente com vogais (Ferreiro \& Teberosky, 1982), omissões de vogais representam a maioria dos erros das crianças que falam inglês (Kamii et al., 1990; Read, 1986; Varnhagen, Boechler, \& Steffler, 1999). Em um estudo, Pollo et al. (2005) confirmaram essas diferenças, mostrando que crianças brasileiras usam mais vogais do que as americanas.

Além disso, foi também testada a hipótese de que tais diferenças refletiriam assimetrias entre os idiomas, tanto em seus sistemas de nome das letras como em seus vocabulários. Pollo et al. (2005) analisaram o vocabulário escrito das crianças por meio de base de dados de livros infantis no Brasil (Pinheiro, 1996) e nos Estados Unidos (Zeno, Ivenz, Millard, \& Duvvuri, 1995) e mostraram que palavras em português têm mais vogais que correspondem ao nome da letra do que em inglês. Palavras em português também têm um maior número de vogais e uma proporção vogal-consoante maior do que as palavras inglesas (e.g, pato tem uma proporção vogal-consoante de 1.0, enquanto sua tradução em inglês duck tem a proporção de 0.33). Desse modo, Pollo et al. (2005) atribuíram a diferença encontrada nas escritas produzidas pelas crianças às discrepâncias entre os dois idiomas.

Além das diferenças quantitativas nos sistemas de nomes das letras, outros padrões podem ser encontrados na escrita das crianças. Tais padrões 
podem ocorrer mesmo antes de elas começarem a escrever fonologicamente. Para demonstrar que a escrita infantil não é uma sequência aleatória de letras, Pollo et al. (2009) selecionaram um grupo de crianças pré-fonológicas em uma amostra de crianças brasileiras e americanas. As escritas dessas crianças mostraram um número de padrões que refletiam, entre outros aspectos, a frequência das letras e digramas (sequência de duas letras) na língua escrita da criança. As crianças pré-fonológicas dos dois países apresentaram escritas que divergiam em aspectos consistentes com sua exposição a diferentes sistemas de escrita. Por exemplo, crianças brasileiras utilizaram mais vogais do que as americanas, e estas tiveram uma tendência maior a repetir letras em suas escritas. Corroborando resultados de um estudo anterior (Pollo et al., 2005), Pollo et al. (2009) não encontraram evidência para os ditos padrões universais, tal qual o estágio silábico, em que as crianças escrevem uma letra por sílaba. De um modo geral, os resultados desse estudo revelam que as escritas não fonológicas não são aleatórias, mas preservam certos padrões da escrita à qual a criança é exposta.

A perspectiva da aprendizagem estatística também fornece uma explicação alternativa para outras afirmações construtivistas. Pollo et al. (2009) investigaram tanto a hipótese da quantidade mínima como a hipótese da variação de letras. Em ambos os casos, as escritas refletem a exposição ao texto escrito ao invés do uso de uma regra rígida, como argumentam os construtivistas. Por exemplo, como explicado anteriormente, existe uma forte correlação entre a extensão das escritas das crianças e o tamanho do texto. Também existe grande relação com as estatísticas do ambiente linguístico, de maneira que as crianças expostas à língua inglesa usam letras duplicadas (e.g. bb e cc) mais frequentemente do que as expostas ao idioma português, refletindo a frequência relativa de letras duplicadas em inglês e em português. Exemplo disso é a palavra comitê em português, e sua equivalente committee em inglês. Em ambos os casos, a perspectiva da aprendizagem estatística parece prover uma explicação mais plausível para a hipótese da quantidade mínima e a da variação de letras.
Os resultados apresentados mostram que diferenças entre idiomas quanto à prevalência de nomes das letras e à frequência de letras e digramas (sequências de duas letras), são fontes de diferenças translinguísticas no desenvolvimento da escrita. Também é de grande importância o entendimento sobre a regularidade do mapeamento entre fonemas e letras.

Mas diferenças entre as línguas quanto à relação letra-som merecem uma avaliação mais precisa do que tem sido feita. Classificações anteriores no sistema de escrita foram elaboradas de forma intuitiva, perguntando-se a pesquisadores em qual dos diferentes níveis de regularidade eles categorizariam sua linguagem escrita (Seymour et al., 2003). Kessler e Treiman (2001) estudaram quantitativamente as relações letra-som em palavras do inglês e concluíram que esse idioma não é tão irregular como fora assumido previamente. Apesar de a ortografia da língua inglesa parecer caótica, muitas regras condicionais do inglês aumentam significativamente sua consistência. Essas regras podem ser mais complicadas do que algumas regras contextuais do português (e.g., $m$, e não $n$, antes de $p$ e b), mas mesmo assim são utilizadas também pelas crianças ao escreverem palavras isoladas (Hayes, Treiman \& Kessler, 2006; Kessler, 2009). Ou seja, estudos têm demonstrado que crianças se beneficiam das regularidades contextuais que o idioma fornece, usando padrões probabilísticos baseados nas estatísticas da língua.

A atenção às propriedades estatísticas da escrita pode predizer o sucesso no aprendizado posterior da escrita. Em um estudo longitudinal com crianças falantes de português, Kessler et al. (2013) testaram crianças da educação infantil, classificadas como pré-fonológicas, com idade média de quatro anos e três meses, e observaram que, quanto mais forte a correlação entre a frequência de digramas (sequência de duas letras) nas escritas das crianças e a frequência de digramas existentes em livros infantis, melhor a nota em um teste padronizado de escrita (Stein, 1994) aplicado dois anos e meio mais tarde no ensino fundamental. Dessa forma, as crianças que tiveram escritas pré-fonológicas que se correlacionaram com as propriedades estatísticas 
do texto obtiveram melhor desempenho, mais tarde, na escrita convencional.

Importantes implicações podem advir de estudos baseados na perspectiva da aprendizagem estatística. O fato de as escritas pré-fonológicas terem padrões que predizem a escrita mais tarde pode ser importante na detecção precoce de crianças em risco de dificuldades na leitura e escrita. A revelação precoce de futuros problemas de leitura e de escrita oferece a possibilidade para uma intervenção antecipada e pode guiar educadores na correção de dificuldades de aprendizado. Ensinar crianças a detectar padrões no texto escrito e, ao mesmo tempo, demonstrar explicitamente alguns desses padrões pode ser uma parte relevante no ensino da leitura e da escrita. Vários padrões são aprendidos tardiamente no desenvolvimento da escrita, bem como muitos padrões não são utilizados tão frequentemente como poderiam (Kessler, 2009). Entretanto, é necessária uma investigação mais pormenorizada acerca do efeito do ensino explícito desses padrões sobre a aprendizagem posterior da escrita.

Por isso, a perspectiva da aprendizagem estatística assegura que as crianças apreendem regularidades estatísticas nas palavras às quais são expostas desde cedo no seu desenvolvimento. Essas regularidades dão às crianças informações sobre padrões gráficos e fonológicos da língua, os quais podem ser detectados até mesmo nas primeiras escritas das crianças. Enfim, o estudo de certos padrões presentes na escrita pode explicar diferenças aparentes entre as escritas de crianças em diversos idiomas.

Concluindo, este artigo revisou três abordagens sobre o desenvolvimento da escrita. A perspectiva fonológica assegura que a compreensão principal no desenvolvimento da escrita é a de que as letras representam os sons das palavras faladas. Uma segunda perspectiva, aqui denominada construtivista, afirma que crianças em sociedades letradas sabem muito sobre a escrita, mesmo antes de entenderem que letras representam fonemas. Mas algumas ideias básicas da perspectiva construtivista, como o estágio silábico, não são confirmadas por estudos rigorosos. Por fim, a última parte deste artigo introduz uma perspectiva relativamente nova - a aprendizagem estatística.

Ela propõe que são as propriedades estatísticas das palavras que influenciam a escrita das crianças, desde o início de seu desenvolvimento. Os estudos discutidos aqui demonstram como as características dos sistemas de escrita influenciam a aprendizagem da escrita. Conclui-se que é crucial entender as características tanto fonológicas como não-fonológicas da escrita infantil e como elas se relacionam com a escrita posterior. Afinal, isso pode auxiliar a identificar crianças em risco de enfrentar dificuldades de alfabetização. Além disso, esse conhecimento também pode ter implicações práticas para o ensino e remediação de dificuldades durante a alfabetização.

\section{Referências}

Besse, J. (1996). An approach to writing in kindergarten. In C. Pontecorvo, M. Orsolini, B. Burge, \& L. Resnick (Eds.), Children's early text construction (pp.127-144). Mahwah, NJ: Erlbaum.

Bissex, G. L. (1980). Gnys at wrk. Cambridge, MA: Harvard University Press.

Caravolas, M. (2004). Spelling development in alphabetic writing systems: A cross-linguistic perspective. European Psychologist, 9(1), 3-14.

Caravolas, M., \& Bruck, M. (1993). The effect of oral and written language input on children's phonological awareness: A cross-linguistic study. Journal of Experimental Child Psychology, 55(1), 1-30.

Cardoso-Martins, C. (2005). Beginning reading acquisition in Brazilian Portuguese. In R. M. Joshi \& P. G. Aaron (Eds.), Handbook of orthography and literacy (pp.171-188). Mahwah, NJ: Erlbaum.

Cardoso-Martins, C., Corrêa, M. F., Lemos, L. S., \& Napoleão, R. F. (2006). Is there a syllabic stage in spelling development? Evidence from Portuguesespeaking children. Journal of Educational Psychology, 98(3), 628-641.

Cardoso-Martins, C., Resende, S. M., \& Rodrigues, L. A. (2002). Letter name knowledge and the ability to learn to read by processing letter-phoneme relations in words: Evidence from Brazilian Portuguese-speaking children. Reading and Writing, 15, 409-432.

Cassar, M., \& Treiman, R. (1997). The beginnings of orthographic knowledge: Children's knowledge of double letters in words. Journal of Educational Psychology, 89(4), 631-644. 
Defior, S., Martos, F., \& Cary, L. (2002). Differences in reading acquisition development in shallow orthographies: Portuguese and Spanish. Applied Psycholinguistics, 23, 135-148.

Ehri, L. C. (1991). The development of reading and spelling in children: An overview. In M. Snowling \& M. Thomson (Eds.), Dyslexia: Integrating theory and practice (pp.63-94). Londres: British Dyslexia Association.

Ehri, L. C. (1998). Grapheme-phoneme knowledge is essential for learning to read words in English. In J. L. Metsala \& L. C. Ehri (Eds.), Word recognition in beginning literacy (pp.3-40). Mahwah, NJ: Erlbaum.

Ellefson, M. R., Treiman, R., \& Kessler, B. (2009). Learning to label letters by sounds or names: A comparison of England and the United States. Journal of Experimental Child Psychology, 102(3), 323-341.

Evans, M. A., \& Saint-Aubin, J. (2005). What children are looking at during shared storybook reading. Psychological Science, 16(11), 913-920.

Ferreiro, E. (1990). Literacy development: Psychogenesis. In Y. Goodman (Ed.), How children construct literacy: Piagetian perspectives (pp.12-25). Newark, DE: International Reading Association.

Ferreiro, E., Pontecorvo, C., \& Zucchermaglio, C. (1996). Pizza or piza? How children interpret the doubling of letters in writing. In C. Pontecorvo, M. Orsolini, B. Burge, \& L. Resnick (Eds.), Children's early text construction (pp.145-163). Mahwah, NJ: Erlbaum.

Ferreiro, E., \& Teberosky, A. (1982). Literacy before schooling. Nova York: Heinemann.

Frith, U. (1985). Beneath the surface of developmental dyslexia. In K. E. Patterson, J. C. Marshall, \& M. Coltheart (Eds.), Surface dyslexia: Neuropsychological and cognitive studies of phonological reading (pp.301-330). Londres: Erlbaum.

Gentry, J. R. (1982). An analysis of developmental spelling in GNYS AT WRK. The Reading Teacher, 36(2), 192-200.

Gough, P. B., \& Hillinger, M. L. (1980). Learning to read: An unnatural act. Bulletin of the Orton Society, 30, 179-196.

Hayes, H., Treiman, R., \& Kessler, B. (2006). Children use vowels to help them spell consonants. Journal of Experimental Child Psychology, 94(1), 27-42.

Henderson, E. (1985). Teaching spelling. Boston: Houghton Mifflin.

Houghton, G., \& Zorzi, M. (2003). Normal and impaired spelling in a connectionist dual-route architecture. Cognitive Neuropsychology, 20(2), 115-162.

Hutzler, F., Ziegler, J. C., Perry, C., Wimmer, H., \& Zorzi, M. (2004). Do current connectionist learning models account for reading development in different languages? Cognition, 91(3), 273-296.

International Phonetic Association. (1999). Handbook of the International Phonetic Association: A guide to the use of the International Phonetic Alphabet. Cambridge: Cambridge University Press.

Kamii, C., Long, R., Manning, M., \& Manning, G. (1990). Spelling in kindergarten: A constructivist analysis comparing Spanish-speaking and English-speaking children. Journal of Research in Childhood Education, 4(2), 91-97.

Kessler, B. (2009). Statistical learning of conditional orthographic correspondences. Writing Systems Research, 1(1), 19-34.

Kessler, B., Pollo, T. C., Treiman, R., \& Cardoso-Martins, C. (2013). Frequency analyses of prephonological spellings as predictors of success in conventional spelling. Journal of Learning Disabilities, 9(4), 317-330. http//:dx. doi.org/10.1177/0022219412449440

Kessler, B., \& Treiman, R. (2001). Relationships between sounds and letters in English monosyllables. Journal of Memory and Language, 44(4), 592-617.

Levin, I., \& Aram, D. (2004). Children's names contribute to early literacy: A Linguistic and a social perspective. In D. Ravid \& H. Bat-Zeev Shyldkrot (Eds.), Perspectives on language and language development (pp.219-239). Dordrecht: Kluwer.

Levin, I., Patel, S., Margalit, T., \& Barad, N. (2002). Letter names: Effect on letter saying, spelling, and word recognition in Hebrew. Applied Psycholinguistics, 23, 269-300.

Liberman, I. Y., Shankweiler, D., Fischer, F. W., \& Carter, B. (1974). Explicit syllable and phoneme segmentation in the young child. Journal of Experimental Child Psychology, 18, 201-212.

Nunes Carraher, T., \& Rego, L. R. B. (1984). Desenvolvimento cognitivo e alfabetização. Revista Brasileira de Estudos Pedagógicos, 63(149), 38-55.

Pacton, S., Perruchet, P., Fayol, M., \& Cleeremans, A. (2001). Implicit learning out of the lab: The case of orthographic regularities. Journal of Experimental Psychology: General, 130(3), 401-426.

Pinheiro, A. M. V. (1996). Contagem de freqüência de ocorrência de palavras expostas a crianças na faixa pré-escolar e séries iniciais do $1^{\circ}$ grau. São Paulo: ABD.

Pollo, T. C., Kessler, B., \& Treiman, R. (2005). Vowels, syllables, and letter names: Differences between young children's spelling in English and Portuguese. Journal of Experimental Child Psychology, 92(2), 161-181.

Pollo, T. C., Kessler, B., \& Treiman, R. (2009). Statistical patterns in children's early writing. Journal of Experimental Child Psychology, 104(4), 410-426.

Pontecorvo, C. (1996). Introduction. In C. Pontecorvo, M. Orsolini, B. Burge, \& L. Resnick (Eds.), Children's early text construction (pp.345-357). Mahwah, NJ: Erlbaum.

Pontecorvo, C., \& Zucchermaglio, C. (1988). Modes of differentiation in children's writing construction. European Journal of Psychology of Education, 3(4), 371-384. 
Read, C. (1986). Children's creative spelling. Londres: Routledge \& Kegan Paul.

Saffran, J. R., Aslin, R. N., \& Newport, E. L. (1996). Statistical learning by 8 -month-old infants. Science, 274(5294), 1926-1928.

Seidenberg, M. S. (1997). Language acquisition and use: Learning and applying probabilistic constraints. Science, 275(5306), 1599-1603.

Seymour, P. H. K., Aro, M., \& Erskine, J. M. (2003). Foundation literacy acquisition in European languages. British Journal of Psychology, 94(Pt 2), 143-175.

Silva, C., \& Alves-Martins, M. (2002). Phonological skills and writing of pre-syllabic children. Reading Research Quarterly, 37(4), 466-483.

Stein, L. M. (1994). TDE: Teste de desempenho escolar: manual para aplicação e interpretação. São Paulo: Casa do Psicólogo.

Treiman, R. (1993). Beginning to spell: A study of first-grade children. Nova York: Oxford University Press.

Treiman, R. (1994). Use of consonant letter names in beginning spelling. Developmental Psychology, 30(4), 567-580.

Treiman, R., \& Broderick, V. (1998). What's in a name: Children's knowledge about the letters in their own names. Journal of Experimental Child Psychology, 70, 97-116.

Treiman, R., \& Kessler, B. (2003). The role of letter names in the acquisition of literacy. In R. Kail (Ed.), Advances in Child Development and Behavior (Vol.31, pp.105-135). San Diego: Academic Press.

Treiman, R., Kessler, B., \& Bourassa, D. (2001). Children's own names influence their spelling. Applied Psycholinguistics, 22(4), 555-570.
Treiman, R., Kessler, B., \& Pollo, T. C. (2006). Learning about the letter name subset of the vocabulary: Evidence from U.S. and Brazilian preschoolers. Applied Psycholinguistics, 27(2), 211-227.

Varnhagen, C. K., Boechler, P. M., \& Steffler, D. J. (1999). Phonological and orthographic influences on children's vowel spelling. Scientific Studies of Reading, 3(4), 363-379.

Vernon, S. A., \& Ferreiro, E. (1999). Writing development: A neglected variable in the consideration of phonological awareness. Harvard Educational Review, 69(4), 395-414.

Wimmer, H., \& Hummer, P. (1990). How German speaking first graders read and spell: Doubts on the importance of the logographic stage. Applied Psycholinguistics, 11(40), 349-368.

Wimmer, H., \& Landerl, K. (1997). How learning to spell German differs from learning to spell English. In C. Perfetti, L. Rieben, \& M. Fayol (Eds.), Learning to spell: Research, theory, and practice across languages (pp.81-96). Mawah, NJ: Erlbaum.

Zacks, R. T., \& Hasher, L. (2002). Frequency processing: A twenty-five year perspective. In P. Sedlmeier (Ed.), Frequency processing and cognition (pp.21-36). Londres: University Press.

Zeno, S. M., Ivenz, S. H., Millard, R. T., \& Duvvuri, R. (1995). The educator's word frequency guide. Brewster, NY: Touchstone Applied Science Associates.

Recebido: janeiro 30, 2013

Versão final: março 7, 2014

Aprovado: abril 2, 2014 
